\title{
Prenatal hyperechoic lung images, a common aspect for multiple diagnoses: retrospective monocentric study
}

\author{
Michela Cing Yu Wong ${ }^{1}$, Valentine FAURE BARDON ${ }^{1}$, Konstantinos FARMAKIS ${ }^{1}$, \\ Laureline BERTELOOT ${ }^{1}$, Alexandre Lapillonne ${ }^{1}$, Christophe DELACOURT ${ }^{1}$, Sabine \\ SARNACKI ${ }^{1}$, Yves VILLE ${ }^{1}$, and Naziha KHEN-DUNLOP ${ }^{1}$ \\ ${ }^{1}$ Necker-Enfants Malades Hospitals
}

May 6, 2020

\begin{abstract}
Objective. Hyperechoic lung images are largely detected prenatally but their underlying etiology is still poorly defined. The aim of the study was to determine the concordance between pre and postnatal diagnosis of prenatal hyperechoic lung images. Design. Retrospective monocentric study Setting. University Hospital of Necker-Enfants malades from January 2009 to December 2018 Population. All fetuses with prenatal hyperechoeic lung images. Methods. Prenatal ultrasound evaluation was performed by a fetal medicine specialist. Postnatal diagnosis was based on CT-scan. Pre- and postnatal features were retrieved from medical charts. Main outcome measures. Accuracy of the prenatal diagnosis in the identification of the malformations and the prediction of postnatal symptoms. Results. 75 patients were included. Main prenatal diagnoses were bronchopulmonary sequestrations (BPS) $(\mathrm{n}=24-32 \%)$, pulmonary cystic malformations (PCM) ( $\mathrm{n}=19-25 \%)$, congenital lobar emphysemas (CLE) $(\mathrm{n}=15-20 \%)$. Mediastinal shift was observed in 18 cases $(24 \%)$; in utero intervention was required in 2. For BPS, the prenatal detection of a systemic arterial supply had a sensitivity of $96 \%$, a specificity of $83 \%$, a PPV of $77 \%$ and an NPV of $98 \%$. For PCM, the prenatal detection of a cystic component had a sensitivity of $69 \%$, a specificity of $69 \%$, a PPV of $67 \%$ and a NPV of 71\%. All 16 neonates with prenatal isolated mediastinal shift were asymptomatic at birth. Seven neonates $(\mathrm{CLE}=5$, BPS $=1$, $\mathrm{BC}=1$ ) showed respiratory distress that were not predicted prenatally. Conclusions. Hyperechoic lung malformations reflect a heterogeneous group of lesions. Symptoms at birth are present in $9 \%$ and cannot be predicted by prenatal features.
\end{abstract}

\section{METHODS}

This is a retrospective study of all consecutive patients diagnosed prenatally with hyperechoic lung images examined at our institution between 2009 and 2018. The study was approved by the institutional review board and no written consent was required from the families.

Information on pre- and post-natal periods and imaging were retrieved from the medical charts. Prenatal evaluation was performed by a fetal medicine specialist and monthly serial ultrasound examination was performed from referral and up until delivery using a Voluson 730, Expert E8 or E10 (General Electric, Zipf, Austria). Each ultrasound examination included description of the mass and its evolution since the last exam: laterality, echogenicity, detection or absence of a feeding systemic arterial supply, and size in the three plans. Fetal hemodynamics was also serially assessed. Presence or absence of complications defined as mediastinal shift, compression of neighboring organs, signs of heart failure, polyhydramnios and hydrops were evaluated.

Gestational age at birth and birth weight were recorded; neonatal and postnatal respiratory signs and symptoms were assessed in all cases. The correlation between prenatal ultrasound features and neonatal symptoms was examined. Postnatal management included confirmation of the prenatal findings by chest 
x-ray in the first days of life and thoracic CT-scan without contrast injection or MRI within the first 6 months of birth.

The need for surgery was assessed by a pediatric surgeon based both on clinical symptoms and CT-scan features. When surgery was required, the histology of the malformation was studied. When conservative management was decided, imaging follow-up was based on CT-scan with low radiation dose. Patients with macrocystic malformation (BC, PCM or hybrid masses) were always referred for surgery, even when asymptomatic. This decision was taken considering the risk of over infection of the cysts, the surgical difficulties after infectious complications and the potential for malignancy. Patients with CLE were operated only when showing respiratory symptoms or signs of compression on follow-up CT-scan. In case of BPS, the surgical indication was based on the presence of secondary clinical symptoms, increase in size of the lesion on follow-up imaging or large systemic arteries.

Data Analysis

Statistical analysis was performed with StatView (Version 5.0). Qualitative variables were expressed as percentages and quantitative variables as mean and standard deviation. Comparison of frequencies of each categorical variable was done by the Fisher's Exact test. All statistical tests were two-sided and p-value lower than 0.05 was considered statistically significant.

\section{RESULTS}

There were 75 cases of hyperechoic prenatal lung images and the mean postnatal follow-up was $3.01 \pm 1.92$ years. Forty-two $(56 \%)$ were boys and $33(44 \%)$ girls. The mean gestational age at first prenatal ultrasound was $23.6 \pm 2.7$ weeks of gestation. Forty-nine cases were left-sided and 26 right-sided.

\section{Prenatal features}

In 28 cases $(37 \%)$ ultrasound examination detected a feeding systemic arterial supply and the proposed diagnosis was BPS in 24 patients and hybrid malformation in 4. Thirteen (46\%) of them showed mediastinal shift, while ascites developed in 1 case at 22 weeks' of gestation (Figure 1 ). Because of the persistence of the ascites, a drain was placed at $28^{\text {th }}$ week of gestation. In this case, postnatal diagnosis was BPS.

In 47 cases $(63 \%)$ no systemic feeding arterial supply was identified prenatally and the diagnosis of other malformations was proposed: 7 BC, 19 PCM, 15 CLE and 6 without defined prenatal diagnosis. Five (11\%) of them had mediastinal shift. In one case, the hyperechoic image was associated with a cystic mass since first ultrasound examination at 14 weeks' of gestation. A compressive BC was suspected with hydropic changes and a cystic-amniotic shunt was placed at 22 weeks' (Figure 2 ). The aspect remained stable until birth at 29 weeks'.

Among all 75 patients, mediastinal shift was observed in 18 cases (24\%). Prenatal procedure was required in two cases because of symptomatic compression, as described above.

The identification of a systemic arterial supply was significantly more associated with mediastinal shift (46\% vs $10 \%, \mathrm{p}=0.0007)$.

\section{Prenatal / neonatal concordance}

For the 75 cases, the final diagnosis based on post-natal CT-scan or post-operative conclusion was BC in 5 cases (7\%), PCM in 5 cases (7\%), hybrid malformations in 10 cases (13\%), BPS in 25 cases (33\%) and CLE in 30 cases $(40 \%)$.

On postnatal evaluation, 35/75 (45\%) patients had a systemic arterial supply, confirming a BPS or a hybrid malformation. Among them, 27 had a prenatal detection of a systemic arterial supply. In one case a systemic arterial supply was described in the prenatal ultrasound, but not confirmed after birth (one patient with CLE). 
The prenatal detection of a systemic arterial supply had a sensitivity of $96 \%$, a specificity of $83 \%$, a positive predictive value of $77 \%$ and a negative predictive value of $98 \%$.

On postnatal evaluation, 37/75 (49\%) patients had a malformation with cystic component: BC, PCM or CLE associated to a bronchocele. Among them, 25 had a prenatal cystic component associated to the hyperechoic image. In 11 cases a cystic component was described in the prenatal ultrasound, but not confirmed after birth (CLE or BPS).

The prenatal detection of a cystic component had a sensitivity of $69 \%$, a specificity of $69 \%$, a positive predictive value of $67 \%$ and a negative predictive value of $71 \%$.

\section{Postnatal outcome}

All 75 fetuses were alive at birth. The mean gestational age at birth was $38.7 \pm 2.1$ weeks and the mean weight at birth was $3208 \pm 486 \mathrm{~g}$.

None of the patients with isolated mediastinal shift developed neonatal symptoms (Figure 3 ). Seven patients (9\%) showed neonatal symptoms with respiratory distress. Two of them had spontaneous resolution and 3 needed urgent intubation. Five of the 7 patients with neonatal symptoms were diagnosed as CLE on the post-natal imaging, one as BC and one with BPS. The symptoms appeared at birth in 5 cases, at day 10 and day 28 in the other 2 cases. The two patients with acute prenatal symptoms developed post-natal respiratory symptoms at birth that lead to neonatal surgery. The patient with prenatal ascites and compression died 4 days after the resection of a thoracic extralobar sequestration, due to uncontrollable pulmonary arterial hypertension. The other patient had an excision of the infected BC and was discharged after 33 days. The details of patients with neonatal symptoms are reported in Table I.

During the long-term follow up, 21 patients (28\%) developed respiratory symptoms: recurrent bronchiolitis or asthma in 14 and pulmonary or bronchial infection in 7 .

The diagnosis of these symptomatic cases was: PCM in 2 cases (9\%), hybrid malformations in 5 cases (24\%), BPS in 9 cases (43\%) and CLE in 5 cases $(24 \%)$.

The details of the symptoms according to the final post-natal diagnosis are described in Table II .

In total, 43 patients $(57 \%)$ were operated. The mean age at surgery was 1.31 years $( \pm 0.73)$.

The histological findings concluded to: 15 BPS (35\%), 10 hybrid malformations (23\%), 5 PCM (12\%), 8 CLE $(18 \%)$ and $5 \mathrm{BC}(12 \%)$ (Figure 4$)$.

At the last review, all the patients were symptom free.

Considering the type of the malformation on the post-natal CT scan, surgery was realized in $5 / 5$ of BC, $5 / 5$ of PCM, 10/10 of hybrid malformations, 15/25 BPS (60\%) and 7/30 (23\%) of CLE.

\section{DISCUSSION}

Hyperechoic lung images on prenatal ultrasound examination form a heterogeneous group of malformations characterized by an increased echogenicity, usually detected by high-resolution ultrasound during the second trimester of pregnancy ${ }^{10}$. Even if this notion is now well accepted, with recent reviews on the subjects ${ }^{13,18}$, very few series are published. Cavoretto at al. ${ }^{9}$ described the largest cohort with 193 cases, in which only two types of malformations were identified before and after birth (congenital cystic airway malformationsCCAM- and BPS). The series of Illanes et al. ${ }^{11}$ used the same classification with 43 CCAM and 5 BPS, but in half of the operated cases the prenatal diagnosis was not confirmed. More recently, Kane et al. added a third group (hybrid malformations) ${ }^{12}$. The confirmation of the diagnosis was good but obtained only for the operated cases (11/13), because no systematic CT scans were performed after birth, leading to an incomplete analysis. In these 3 series no case of CLE was reported, whereas it is now recognized as one of the main causes of prenatal hyperechoic images ${ }^{19,20}$. Based on a systematic post-natal imaging, our series shows in 
detail that various congenital lung malformations can have a prenatal hyperechoic aspect: CLE, PCM, BC, BPS and hybrid malformation.

Microcystic images were not strongly associated to post-natal cystic images, with sensitivity and a specificity of about $70 \%$. On the other hand, the prenatal detection of a systemic arterial supply had a sensitivity of $96 \%$ and a specificity of $83 \%$. Then, while the prenatal diagnosis of a BPS is certain, based on the association of a hyperechoic image and a systemic artery ${ }^{2,9}$, the presence of a hyperechoic zone without the visualization of a systemic artery can lead to various final diagnosis.

Even though compressive signs are exceptional, they can be considered as an indication for a prenatal procedure. In our two cases, they were diagnosed early, at 22 weeks', according to the published previous cases, stressing the importance of the $2^{\text {nd }}$ trimester evaluation and a close ultrasound monitoring ${ }^{12}$. For all these reasons, we propose a cautious prenatal approach for hyperechoic images, avoiding the term of microcystic that appears confusing and strong enough, and focusing on the morphological description of the images and the local consequences.

Concerning the associated prenatal features, mediastinal shift was frequently observed ( $1 / 4$ of our patients), especially in those with BPS. In agreement with previous series, mediastinal shift was not associated to higher risk of neonatal symptoms and, when isolated, it should not be interpreted as a sign of increased morbidity ${ }^{21-23}$. Moreover, among the 7 patients with neonatal symptoms, 5 had no compression signs on the prenatal ultrasound and the final postnatal diagnosis was variable (cf Table 1). These data lead us to think that neonatal respiratory failure or secondary symptoms cannot be anticipated by the prenatal evaluation. Congenital lung malformations should thus be referred to an expert perinatal team to organize the neonatal management.

Systematic post-natal imaging showed that all the malformations were present after birth and during the 3 years mean follow-up. Even though few cases of complete regression of lung malformations have been described and well-documented, it has to be considered as an exceptional event and should not be strengthened during the prenatal counseling. Recent evaluations have shown that the changes in the lung echogenicity in the $3^{\text {rd }}$ trimester (or signal intensity on prenatal MRI) could be the physiological explanation of the apparent regression, reported in the historical series ${ }^{24}$. All cases with abnormal echogenicity of the lungs should be controlled after birth, even in cases of disappearance on the late US evaluations.

According to our practice, close to $60 \%$ of the patients were operated. The natural history of congenital lung malformations varies widely from asymptomatic cases to severe cardiopulmonary symptoms. Whereas there is an agreement for the surgery of all the symptomatic lung malformations, the management of asymptomatic patients is still controversial ${ }^{25,26}$. Some clinicians would advise the excision of the malformation, while others could argue that the risks are not high enough to warrant subjecting the child to a major operation and its potential complications ${ }^{27,28}$. Apart from the risk of pulmonary infections, the risk of malignancy remains the most worrying factor for surgeons who recommend prophylactic excision of asymptomatic patients with cystic components (PCM and BC), that is our policy ${ }^{29-32}$. CLE and BPS were thus treated conservatively with secondary symptoms during the follow-up period twice more frequent in BPS.

This large series confirms that the ability to correctly defined congenital lung malformations by prenatal ultrasound is limited ${ }^{18}$, except when a hyperechoic image is visualized in association with a systemic artery that could highly predict a BPS. Prenatal imaging should then mainly focused on the description of the images and the local consequences more than trying to name a malformation.

\section{DECLARATIONS}

Ethics statement

The study was approved by the institutional review board.

Name of ethics committee: CENEM. Date: 28/10/2019. Ref: 2019-23-MW. 
Funding . This research did not receive any specific grant from funding agencies in the public, commercial, or not-for-profit sectors.

Conflicts of interest. The authors declare that they have no conflict of interest.

Contribution to Authorship. NKD is responsible of the study design. MW and FK collected the prenatal and postnatal findings. MW is responsible for the statistical analysis and she wrote down the proof of the manuscript. VFB, BL, AL, CD, YV, SS and NKD made major revisions of the manuscript.

\section{REFERENCES}

1. Colon N, Schlegel C, Pietsch J, Chung DH, Jackson GP. Congenital lung anomalies: can we postpone resection? J Pediatr Surg. 2012 Jan; 47(1):87-92.

2. Durell J, Lakhoo K. Congenital cystic lesions of the lung. Early Hum Dev. 2014 Dec; 90(12):935-9.

3. Hall NJ, Stanton MP. Long-term outcomes of congenital lung malformations. Semin Pediatr Surg. 2017 Oct;26(5):311-316.

4. Benachi A. Echogenic fetal lung lesions. Ultrasound Obstet Gynecol. 2008 Nov;32(6):729-31.

5. Cass DL, Crombleholme TM, Howell LJ, Stafford PW, Ruchelli ED, Adzick NS. Cystic lung lesions with systemic arterial blood supply: a hybrid of congenital cystic adenomatoid malformation and bronchopulmonary sequestration. J Pediatr Surg. 1997 Jul;32(7):986-90.

6. MacKenzie TC, Guttenberg ME, Nisenbaum HL, Johnson MP, Adzick NS. A fetal lung lesion consisting of bronchogenic cyst, bronchopulmonary sequestration, and congenital cystic adenomatoid malformation: the missing link? Fetal Diagn Ther. 2001 Jul-Aug;16(4):193-5.

7. Langston C. New concepts in the pathology of congenital lung malformations. Semin Pediatr Surg. 2003 Feb;12(1):17-37.

8. Adzick NS. Management of fetal lung lesions. Clin Perinatol. 2009 Jun;36(2):363-76, x.

9. Cavoretto P, Molina F, Poggi S, Davenport M, Nicolaides KH. Prenatal diagnosis and outcome of echogenic fetal lung lesions. Ultrasound Obstet Gynecol. 2008 Nov;32(6):769-83.

10. Baird R, Puligandla PS, Laberge JM. Congenital lung malformations: informing best practice. Semin Pediatr Surg. 2014 Oct;23(5):270-7.

11. Illanes S, Hunter A, Evans M, Cusick E, Soothill P. Prenatal diagnosis of echogenic lung: evolution and outcome. Ultrasound Obstet Gynecol. 2005 Aug;26(2):145-9.

12. Kane SC, Da Silva Costa F, Crameri JA, Reidy KL, Kaganov H, Palma-Dias R. Prenatal assessment and postnatal outcome of fetal echogenic lung lesions: a decade's experience at a tertiary referral hospital. J Matern Fetal Neonatal Med. 2019 Mar;32(5):703-709.

13. Degenhardt J, Kohl T, Enzensberger C, Axt-Fliedner R. Review on current management and outcome data of echogenic lung lesions and hydrothorax of the fetus. Z Geburtshilfe Neonatol. 2013 Dec;217(6):204-10.

14. Kini H, Sreeram S, Shukla S, Rao S, Sahu K, Adiga D, Suresh P. Congenital Pulmonary Airway Malformation - 19-Year Experience from a Tertiary Care Center in India. Fetal Pediatr Pathol. 2019 May 14:1-11.

15. Stocker JT, Madewell JE, Drake RM. Congenital cystic adenomatoid malformation of the lung. Classification and morphologic spectrum. Hum Pathol. 1977 Mar;8(2):155-71.

16. Stocker JT. The respiratory tract. In Pediatric Pathology (2nd edn), Stocker JT, Dejner LP (eds). Lippincott Williams \& Wilkins: Philadelphia, PA, 2001; 466-473. 
17. Adzick NS, Harrison MR, Glick PL, Golbus MS, Anderson RL, Mahony BS, Callen PW, Hirsch JH, Luthy DA, Filly RA, et al. Fetal cystic adenomatoid malformation: prenatal diagnosis and natural history. J Pediatr Surg. 1985 Oct;20(5):483-8.

18. Achiron R, Hegesh J, Yagel S. Fetal lung lesions: a spectrum of disease. New classification based on pathogenesis, two-dimensional and color Doppler ultrasound. Ultrasound Obstet Gynecol. 2004 Aug;24(2):107-14.

19. Oliver ER, DeBari SE, Horii SC, Pogoriler JE, Victoria T, Khalek N, Howell LJ, Adzick NS, Coleman BG. Congenital Lobar Overinflation: A Rare Enigmatic Lung Lesion on Prenatal Ultrasound and Magnetic Resonance Imaging. J Ultrasound Med. 2019 May;38(5):1229-1239.

20. Johnston JH, Kline-Fath BM, Bitters C, Calvo-Garcia MA, Lim FY. Congenital overinflation: prenatal MRI and US findings and outcomes. Prenat Diagn. 2016 Jun;36(6):568-75.

21. Miller JA, Corteville JE, Langer JC. Congenital cystic adenomatoid malformation in the fetus: natural history and predictors of outcome. J Pediatr Surg. 1996 Jun;31(6):805-8.

22. Bunduki V, Ruano R, da Silva MM, Miguelez J, Miyadahira S, Maksoud JG, Zugaib M. Prognostic factors associated with congenital cystic adenomatoid malformation of the lung. Prenat Diagn. 2000 Jun;20(6):45964.

23. Walker L, Cohen K, Rankin J, Crabbe D. Outcome of prenatally diagnosed congenital lung anomalies in the North of England: a review of 228 cases to aid in prenatal counselling. Prenat Diagn. 2017 Oct;37(10):1001-1007.

24. Kunisaki SM, Ehrenberg-Buchner S, Dillman JR, Smith EA, Mychaliska GB, Treadwell MC. Vanishing fetal lung malformations: Prenatal sonographic characteristics and postnatal outcomes. J Pediatr Surg. 2015 Jun;50(6):978-82.

25. Colon N, Schlegel C, Pietsch J, Chung DH, Jackson GP. Congenital lung anomalies: can we postpone resection? J Pediatr Surg. 2012 Jan;47(1):87-92.

26. Morini F, Zani A, Conforti A, van Heurn E, Eaton S, Puri P, Rintala R, Lukac M, Kuebler JF, Friedmacher F, Wijnen R, Tovar JA, Pierro A, Bagolan P. Current Management of Congenital Pulmonary Airway Malformations: A "European Pediatric Surgeons' Association" Survey.

27. Singh R, Davenport M. The argument for operative approach to asymptomatic lung lesions. Semin Pediatr Surg 2015; 24: 187-95.

28. Stanton M. The argument for a nonoperative approach to asymptomatic lung lesions. Semin Pediatr Surg 2015; 24: 183-86.

29. Casagrande A, Pederiva F. Association between congenital lung malformations and lung tumors in children and adults: a systematic review. J Thorac Oncol 2016; 11: 1837-45.

30. Wong KKY, Flake AW, Tibboel D, Rottier RJ, Tam PKH. Congenital pulmonary airway malformation: advances and controversies. Lancet Child Adolesc Health. 2018 Apr;2(4):290-297.

31. Kanemitsu Y, Nakayama H, Asamura H, Kondo H, Tsuchiya R, Naruke T. Clinical features and management of bronchogenic cysts: report of 17 cases. Surg Today. 1999;29(11):1201-5.

32. Gómez-Hernández MT1, Novoa N2, Aranda JL2, Jiménez-López MF2. Enteric Adenocarcinoma Arising from a Bronchogenic Cyst. Arch Bronconeumol. 2017 Sep;53(9):523-524.

TABLES

Table I. Antenatal details of patients with neonatal symptoms.

\begin{tabular}{llllll}
\hline Antenatal & Antenatal & Antenatal & Neonatal symptoms* & Neonatal symptoms* & Neona \\
\hline Mediastinal shift & Systemic supply & Prenatal procedure & Birth term & Birth weight & Days a
\end{tabular}




\begin{tabular}{lllllll}
\hline & Antenatal & Antenatal & Antenatal & Neonatal symptoms* & Neonatal symptoms* & Neona \\
\hline Case 1 & No & No & No & 41 & 3940 & 28 \\
Case 2 & No & No & No & 38 & 3315 & 10 \\
Case 3 & No & No & No & 41 & 3300 & 0 \\
Case 4 & No & No & No & 35 & 3220 & 0 \\
Case 5 & No & No & No & 39 & 2950 & 0 \\
Case 6 & Oui & Oui & Oui & 38 & 1660 & 0 \\
Case 7 & No & Oui & Oui & 30 & & 0 \\
\hline
\end{tabular}

$\mathrm{CLE}=$ Congenital lobar emphysema, $\mathrm{BPS}=$ bronchopulmonary sequestration, $\mathrm{BC}=$ bronchogenic cyst

* Respiratory distress, shortness of breath, intercostal retractions, desaturation.

Table 2: Incidence of symptoms according to the final diagnosis

\begin{tabular}{lllll}
\hline Final Diagnosis & Number of patients & Antenatal Symptoms & Neonatal Symptoms & Postnatal Symptoms * \\
\hline BC & 5 & $1(20 \%)$ & $1(20 \%)$ & $0(0 \%)$ \\
PCM & 5 & $0(0 \%)$ & $0(0 \%)$ & $2(40 \%)$ \\
Hybrid & 10 & $3(30 \%)$ & $0(0 \%)$ & $5(50 \%)$ \\
BPS & 25 & $10(12 \%)$ & $1(4 \%)$ & $9(36 \%)$ \\
CLE & 30 & $4(13 \%)$ & $5(17 \%)$ & $5(17 \%)$ \\
\hline
\end{tabular}

$\mathrm{BC}=$ bronchogenic cyst, $\mathrm{PCM}=$ pulmonary cystic malformation, $\mathrm{BPS}=$ bronchopulmonary sequestration, $\mathrm{CLE}=$ Congenital lobar emphysema.

* Recurrent bronchiolitis, asthma, pulmonary or bronchial infection. 

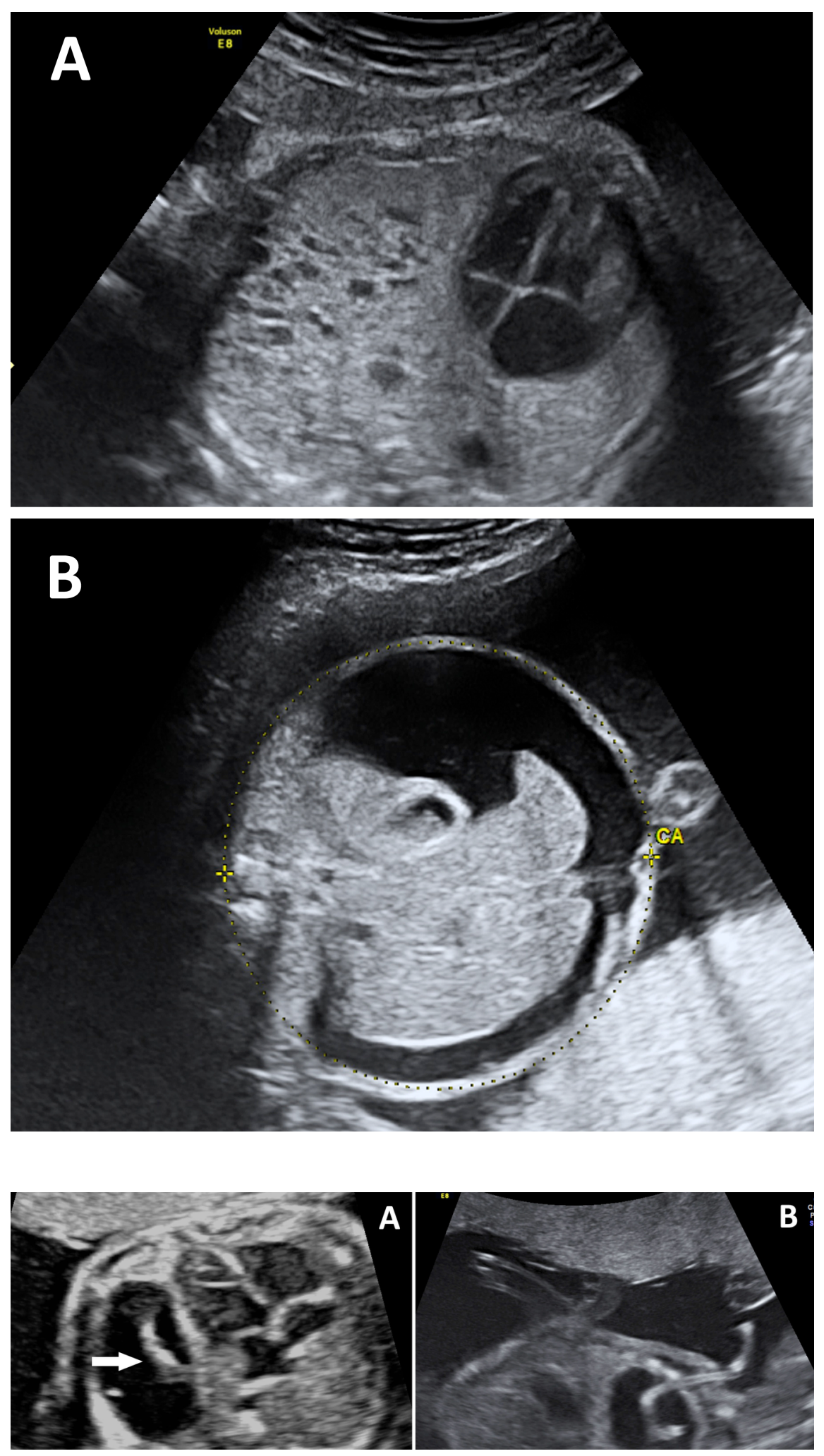

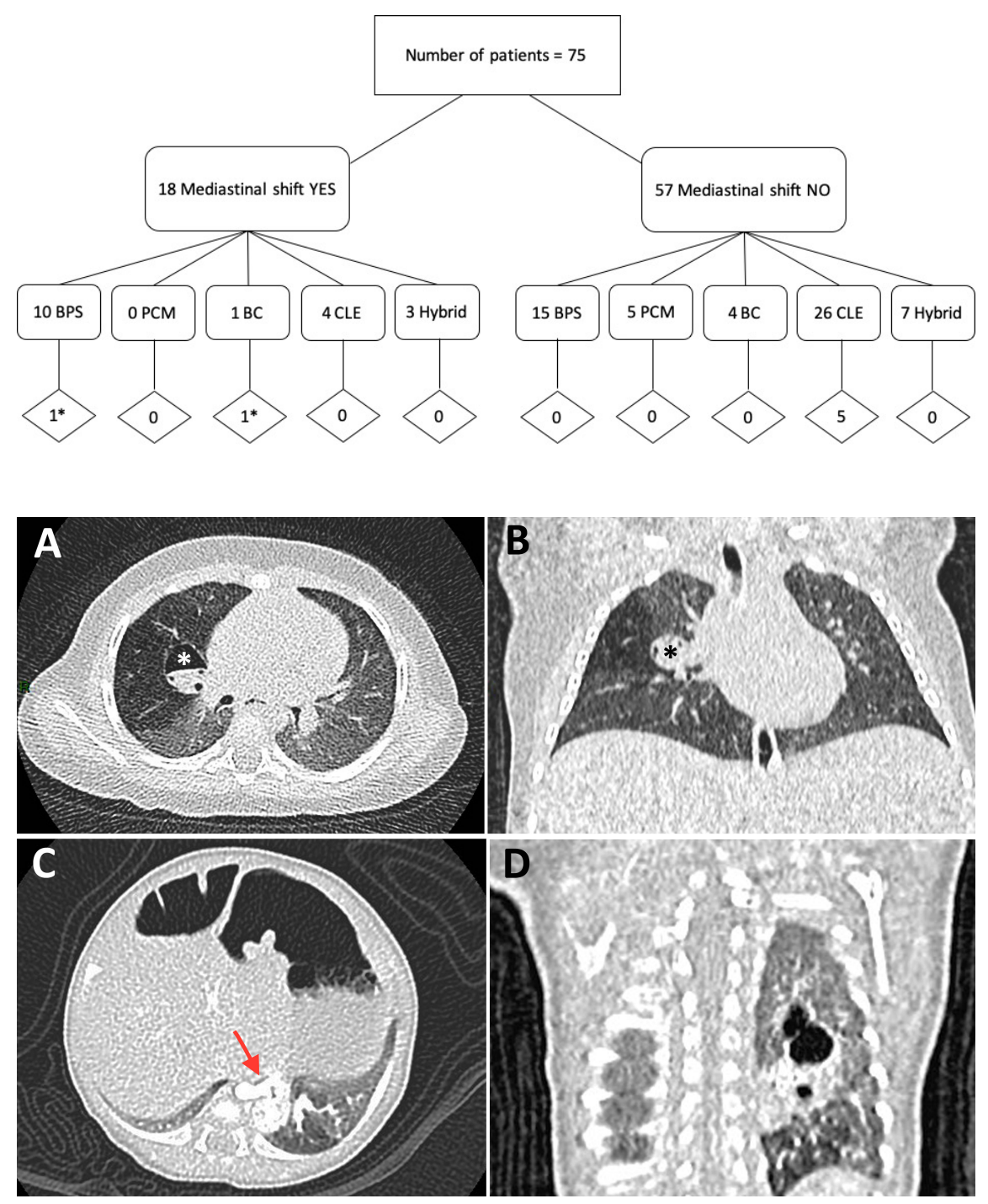